\title{
Almacenamiento prehispánico de una aldea maya: análisis de material cerámico procedente de Joya de Cerén, El Salvador
}

Prehispanic storage system of a mayan town: an analysis of ceramic material from Joya de Cerén, El Salvador

\author{
Michelle Toledo' \\ Stephanie Aguilar ${ }^{2}$ \\ Gabriel Lozano ${ }^{3}$ \\ Laura Sermeño ${ }^{4}$ \\ Universidad Tecnológica de El Salvador \\ michelletoledo86@gmail.com
}

Recibido: 21/07/17- Aceptado: 18/10/17

\section{Resumen}

El sitio arqueológico Joya de Cerén, ubicado en el municipio de San Juan Opico, departamento de La Libertad, fue descubierto de manera accidental en 1976 y declarado como patrimonio de la humanidad por la Unesco en 1993. En el lugar se encuentran vestigios de la vida cotidiana y de las actividades agrícolas, sociales y religiosas de una pequeña comunidad maya sepultada por una erupción volcánica alrededor del año 650 d.C. Hasta el momento se ha discutido poco sobre aspectos organizativos del sitio, como el modo de abastecimiento o almacenamiento prehispánico, por tal razón, en esta investigación se pretende dar un acercamiento sobre el análisis e interpretación de materiales cerámicos relacionados con los modos de producción agrícola y los aspectos mencionados; y así crear nuevos paradigmas sobre actividades humanas, en especial en relación con los pueblos originarios de El Salvador.

\section{Palabras clave}

Sitios arqueológicos - El Salvador; Arqueología e historia El Salvador; Joya de Cerén - Patrimonio de la humanidad

\section{Abstract}

Joya de Cerén, an archaeological site in the municipality of San Juan Opico, Department of La Libertad, was accidentally discovered in 1976 and declared World Heritage Site by the UNESCO in 1993. This site contains remains of the daily life and agricultural, social and religious activities of a small Mayan community that was buried after a volcanic eruption around 650 A.D. Little has been discussed so far about the organizational aspects of the site such as the prehispanic storage and supply system; consequently, this research aims to provide a closer look at the analysis and interpretation of ceramic materials related to agricultural production methods thus giving way to new paradigms over human activities, particularly those in relation to the indigenous peoples of El Salvador.

\section{Keywords}

Archaeological sites - El Salvador; Archaelogy and history - El Salvador; Joya de Cerén - World heritage

\footnotetext{
Arqueóloga y administradora del Parque Arqueológico Joya de Cerén, técnica del Departamento de Arqueología en Secultura y docente hora-clase de la asignatura Análisis e Interpretación de Materiales Culturales, Utec.

2 Estudiante de la Licenciatura en Arqueología, Utec.

3 Estudiante de la Licenciatura en Arqueología, Utec.

${ }^{4}$ Estudiante de la Licenciatura en Arqueología, Utec.
} 


\section{Introducción}

El sitio arqueológico Joya de Cerén está ubicado a $35 \mathrm{~km}$ al noroeste de la capital, San Salvador, en el municipio de San Juan Opico, departamento de La Libertad. Fue descubierto de manera accidental en 1976, debido a la nivelación de terrenos con un buldócer para la construcción de silos de almacenamiento de granos del extinto Instituto Regulador de Abastecimiento (IRA). Por el extraordinario estado de conservación en el que se encontraron sus vestigios, se pensó que se trataba de viviendas recientes, aunque esto fue descartado por el aparecimiento de material arqueológico perteneciente al período clásico tardío.

Posterior a su descubrimiento se realizaron las primeras excavaciones en 1978 y continuaron hasta 1994. Actualmente, este importante sitio arqueológico muestra la vida cotidiana de una aldea maya del período clásico tardío (650 d. C.). Además retrata la complejidad de las comunidades mesoamericanas y sus avanzados conocimientos en arquitectura, agricultura, domesticación de animales y plantas, almacenamiento, actividades rituales y comunitarias, uso de espacios y relación con las poblaciones cercanas e intercambios culturales.

Declarado el 11 de diciembre de 1993 como Patrimonio de la Humanidad por la Unesco, Joya de Cerén es hasta el momento el único sitio arqueológico con esta categoría en El Salvador, y uno de los parques arqueológicos más visitados por turistas nacionales e internacionales. Además, es considerado como un icono y referencia en el mundo arqueológico.

Aunque existen muchos trabajos multidisciplinarios de investigación sobre el sitio, muy poco se ha hablado de sus actividades como grupo social o como unidades domésticas. Asimismo, se ha indagado poco sobre sus contextos con el entorno natural como de las actividades agrícolas, de producción y almacenaje, dejando vacíos de interpretación que podrían ayudar a comprender la vida cotidiana, pero a la vez compleja, de una aldea maya de dicho período arqueológico.

Con esta investigación se pretende dar un acercamiento sobre el análisis e interpretación de materiales cerámicos relacionados con los modos de almacenamiento prehispánico en una aldea maya procedentes de las estructuras prehispánicas con funciones de bodegas de este importante sitio arqueológico y así descubrir o crear nuevos paradigmas sobre actividades humanas, en especial, de los pueblos originarios de El Salvador.
Almacenamiento prehispánico: subsistencia, abastecimiento y poder

Luego del surgimiento del sedentarismo a consecuencia de la agricultura, en los pueblos prehispánicos se vieron por primera vez reservas de alimentos mayores que las que obtenían mediante la recolección y la caza. A pesar de esto, se sabe que antes del surgimiento de la agricultura hubo una fase de almacenamiento de alimentos perecederos, los cuales servían como reserva para los períodos más difíciles de recolección.

Cuando hablamos de almacenar nos referimos a diferentes aspectos como reunir, guardar o registrar en cantidad materiales, ya sean de diferentes tipos o de uno determinado. Por tal razón, la acción de almacenar conlleva diferentes actividades que pueden ser estudiadas, tanto antropológica como arqueológicamente, con base en los contextos arqueológicos y los materiales recolectados para análisis.

Según Hirth (2012), los sistemas de almacenamiento varían y se complejizan según avancen las culturas en sus modos de producción. El tipo de almacenamiento está íntimamente relacionado con el tiempo en el cual vaya a consumirse el producto, que puede variar entre el consumo inmediato (desde su recolecta hasta pocas horas después del procesamiento del recurso) o reservar lo obtenido y utilizarlo días, semanas o incluso meses después.

Es por esto que el estudio de esta actividad en la arqueología nos brinda importante información sobre la complejidad social que tiene determinado grupo, es decir, una unidad doméstica tendrá modos de recolección y abastecimiento menos complejos que un centro ceremonial, en el cual incluso esta actividad puede ser considerada como tributo.

Como lo menciona Hirth (2012), un aspecto importante del almacenamiento es su carácter cultural, en el cual podemos comprender cómo a través de esta práctica se garantiza un aumento en las posibilidades de supervivencia. Asimismo, destaca la importancia desde el punto de vista doméstico, como nivel más elemental de la sociedad, y el institucional (centros urbanos o ceremoniales), en el cual sus objetivos de reducción de riesgo, proyección social y economía política, siendo estos parte de una "inversión" como beneficio social y económico en el almacenamiento. 
Entonces podemos distinguir tanto un almacenamiento doméstico como uno institucional, donde el primero está relacionado con recursos de nivel familiar, que está orientado a la producción de alimentos para asegurar la supervivencia del hogar, reduciendo los riesgos de subsistencia y ser una estrategia de economía doméstica para los momentos en los cuales hay escases de recursos, ya sea por factores ambientales o indirectos. En el segundo, el almacenamiento institucional está asociado con las estrategias económicas de estos bienes para sostener la élite social y las actividades sociales, donde a mayor abastecimiento será mayor el poder y el grado de supervivencia.

De acuerdo con Hirth (2012), existen tres modelos teóricos para comprender el almacenamiento de sociedades antiguas:

\section{Almacenamiento contra los riesgos de subsistencia, proyección social y economía política}

Cuando se habla de almacenamiento contra los riesgos de subsistencia implica varias estrategias a las que recurren los grupos domésticos para protegerse de dichos riesgos, entre las que están las siguientes:

- La movilización hacia un espacio físico donde los recursos son más abundantes.

- La diversificación de recursos.

- El intercambio de recursos por otros a los cuales no se tiene acceso.

- El almacenamiento para un consumo posterior de los recursos.

En este sentido, el uso que le dé un grupo doméstico, por ejemplo, determinará sus estrategias contra los riesgos de subsistencia. Asimismo, no solo se puede hablar de recursos perecederos y no perecederos, podemos hablar incluso de un almacenamiento "vivo" relacionado con la domesticación de animales, que pueden incluso ser alimentados con los recursos resguardados, siendo esto también una estrategia de subsistencia.

El almacenamiento como proyección social, en cambio, se refiere a que el almacenaje es un motor del capital social y de las jerarquías al interior de la sociedad. Esto quiere decir que, dependiendo de los recursos que un grupo posea (objetos suntuosos), le otorgará un estatus dentro de la sociedad porque tendrá un control sobre acumulación de bienes y riquezas. Junto con esto, el tamaño y la localización de las instalaciones de almacenamiento en casas o lugares clave dentro de las comunidades constituyen factores importantes para la influencia social potencial del líder (Hirth, 2012:16).

Según esto, en las comunidades de Cahokia, Estados Unidos, el almacenamiento por medio de agujeros subterráneos, descentralizados y para uso privado era un desafío para la subsistencia de los grupos élites. En cambio, los almacenamientos centralizado y aéreo fueron especialmente comunes en el gran centro ceremonial de Cahokia. Mientras tanto la comunidad se alejaba del centro ceremonial, el uso de almacenamiento subterráneos es más recurrente (Hirth, 2012:17). Asimismo, según Manzanilla (2012), para el período clásico en Teotihuacan, México, en el área conocida como Teopancazco, ubicada al sureste del centro urbano, durante la fase Xolalpan, se constató la presencia de profundas fosas de almacenamiento, asociadas con áreas domésticas e intermedias. Pero para la misma fase, en el área conocida como Oztoyahualco se registraron cuartos para almacenamiento de alimentos y plantas medicinales; yacían contiguo a cocinas. De acuerdo con Manzanilla, el almacenamiento en vasijas hace fácil su traslado y distribución; y en cuanto a la distribución de vajillas finas de servicio, cazuelas y cráteres Miccaotli cerca de la ciudad: que estuviesen relacionados con la preparación y almacenamiento de alimentos para los festejos y comidas comunales de los individuos de alto estatus (Manzanilla, 2012:78).

El almacenamiento, como instrumento de inversión y de economía política, es relacionado directamente con sociedades políticamente organizadas, donde juega un papel importante en el crecimiento de su influencia e interacción con otros pueblos. Ahora los bienes de consumo no son reservados para el uso familiar o del grupo social; sirve como canal de interacción con otras sociedades. El poseer gran cantidad de recursos representa poder económico. El intercambio de bienes toma auge y la preservación de los productos es más especializada, esto con el fin de asegurar la durabilidad de los bienes almacenados. En este modelo se habla de "inversión", refiriéndose a la obtención de nuevos recursos a cambio de los propios. Los bienes de prestigio suelen ser comercializados debido a su gran valor y transpirabilidad.

Una problemática que enfrenta el almacenamiento, y de aquí que las tecnologías desarrolladas para dicho fin sean 
diversas y especializadas, son las condiciones y composición de los bienes que se han de almacenar, teniendo en cuenta si lo que desea preservarse se corrompe de forma rápida o si, por el contrario, no sufre cambios a lo largo del tiempo; también depende del momento en el que se planea tener acceso a estos bienes acumulados.

A través del estudio de los bienes almacenados podemos conocer tanto los sistemas como las tecnologías para su almacenamiento de, así como los productos más utilizados entre los antiguos pobladores de la región y también los diferentes usos y procesamientos de los alimentos conservados.

Para la identificación de los medios de almacenamiento en el registro arqueológico es necesaria la implementación de enfoques etnoarqueológicos, ya que estos en algunos casos logran comparar tecnologías de almacenamiento público en la actualidad que se asemejan a las estructuras de almacenamiento de períodos prehispánicos (Uruñuela et al., 2012).

Algunas de las formas para conservar alimentos en las culturas prehispánicas del norte de México brindan ejemplos análogos aplicables a buena parte del área mesoamericana, estos son presentados por Guevara (2012). Algunos factores de interés para controlar, por los indígenas, fueron humedad, temperatura y cantidad de alimentos almacenados.

Podemos suponer que una de las principales herramientas para almacenar y proteger los alimentos de los factores antes mencionados debieron ser los depósitos cerámicos, que eran capaces de mantener una temperatura constante estando en aislamiento y poseían capacidades impermeables. La versatilidad de formas y tamaños hizo, de estos, parte de una tecnología fundamental para el resguardo de granos, grasas y la indispensable agua.

Estudios sobre los residuos contenidos en la cerámica encontrada en contextos arqueológicos son herramientas de primera mano para el análisis y la interpretación de la función de la cerámica como un espacio básico y fundamental para la protección y el procesamiento de los alimentos y granos destinados a su posterior cultivo, para el consumo y, por ende, para la subsistencia de las sociedades.

Como hemos recalcado, el estudio del almacenamiento a través de la arqueología, que es un enfoque poco explorado, aun siendo un buen exponente de actividad social, pues, de acuerdo con el registro de los restos materiales culturales que antes hemos mencionado (fosas subterráneas, cerámica, restos orgánicos, etc.), podemos comprender técnicas de almacenamiento, estrategias de subsistencia, el almacenamiento como proyección social y política, su complejidad social determinada por su elemento esencial, unidades domésticas y a gran escala como un almacenamiento institucional; todos estos factores nos brindan datos importantísimos para comprensión de nuestros pueblos originarios.

\section{Almacenamiento prehispánico en el sitio arqueológico Joya de Cerén}

El sitio arqueológico Joya de Cerén es poseedor de una cantidad de información incalculable relacionada con actividades cotidianas de una aldea maya del período clásico tardío, esto sumado al hecho de haber sido sepultada por ceniza luego de una erupción volcánica local, dejando encapsulada toda esa valiosa información, elementos materiales esenciales para los estudios arqueológicos, encaminados a descubrir la complejidad de nuestras sociedades ancestrales.

Joya de Cerén se localiza dentro del Valle de Zapotitán, en la carretera que conduce al municipio de San Juan Opíco, departamento de La Libertad. Se encuentra aproximadamente a $450 \mathrm{~m}$ sobre el nivel del mar, donde al este corre el río Sucio, el cual en su cuenca colinda no solamente con Joya de Cerén, sino también los sitios arqueológicos de San Andrés y Las Marías.

Desde ese momento, el sitio arqueológico Joya de Cerén ha estado en continua investigación y ha sido objeto principalmente de estudios de conservación, ya que estas estructuras prehispánicas se encuentran hechas de tierra, es decir, un sistema constructivo de bajareque y tapia, o tierra apisonada, lo cual las hace más frágiles.

A partir de 1978 se comenzaron los trabajos de excavación a cargo del Dr. Payson Sheets, de la Universidad de Colorado, arrojando los primeros datos que determinaban el fechado del sitio para el $590 \pm 90$ d. C. Para los años de 1979 y 1980 se intensifican las investigaciones, incluyendo sondeos geofísicos, principalmente de radar de penetración de suelos, de resistividad, de refracción sísmica y de inducción electromagnética con el objetivo de detectar anomalías en el suelo, las cuales podrían tratarse de otras 
estructuras. Para esos años se identificaron las estructuras 2 y 4 . No fue sino hasta el año de 1989 que se encuentra la estructura 3, y en 1994 se identificaron las estructuras 12 , $10,11,6,7$ y 9 . Siendo realizados en estos años los trabajos de excavaciones más importantes e intensivos para la interpretación del sitio arqueológico.

Debido a su buen estado de conservación y a la importancia arqueológica que posee este sitio, pues evidencia la vida cotidiana de una pequeña comunidad del $650 \mathrm{~d}$. C., ubicándola cronológicamente en el período clásico tardío, que demuestra de manera única la arquitectura de tierra, agricultura, complejos comunitarios, áreas de almacenamiento, entre otras actividades, en 1992, el Consejo Nacional para la Cultura y el Arte (hoy Secretaría de Cultura de la Presidencia) inició las gestiones para que se nombrara a Joya de Cerén como patrimonio mundial. La propuesta fue aceptada por la Unesco en 1993. En reconocimiento de su importancia, el 11 de diciembre de 1993 el sitio Joya de Cerén fue inscrito en la Lista del Patrimonio Mundial de la Unesco; y es el único sitio con esta categoría en El Salvador.

Las últimas excavaciones en Joya de Cerén han sido enfocadas a comprender las dinámicas relacionadas con la agricultura del sitio. Curiosamente, durante estos últimos estudios, siempre dirigidos por el Dr. Sheets, fue descubierto un camino, o sacbé, que divide algunos surcos de cultivo y que posee una orientación oeste-este, además de que en este se registraran huellas humanas en dirección esteoeste, sugiriendo de cierta manera la ruta de evacuación o de escape al momento de la erupción, justamente en dirección al sitio arqueológico de San Andrés.

El Parque Arqueológico Joya de Cerén, en la actualidad, cuenta con cuatro áreas o grupos arquitectónicos dentro del sitio, siendo el Complejo A constituido por las estructuras prehispánicas 1, 6, 11, 10 y 12; el Complejo B, por las estructuras 2,7 y 9 ; y los complejos $C$ y $D$, por las estructuras prehispánicas 3 y 4 , respectivamente, totalizando diez estructuras prehispánicas excavadas, aunque en el Parque existan por lo menos ocho estructuras más por investigar.

Cabe recalcar que 21 años de investigación arqueológica en el sitio, con un enfoque multidisciplinario, han brindado mucha información sobre la vida cotidiana de esta aldea, aunque la mayoría queda de forma descriptiva y no interpretativa. Algunos de estos estudios han demostrado que Joya de Cerén posee entre sus datos más sobresalientes: edificaciones con funciones específicas; sistemas constructivos: arquitectura (bajareque y tierra apisonada); áreas de cultivo: agricultura; alimentación y almacenamiento (viviendas y bodegas); actividades rituales (casa de la shamana y baño sauna); uso de espacios: posibles unidades domésticas y plaza; cerámica y lítica características del sitio y evidencia del impacto de un fenómeno natural (erupción volcánica) sobre las viviendas, asimismo, que dicho fenómeno probablemente alertó del desastre natural a la población de esta aldea; y por esta razón hasta el momento no se han registrado evidencias de restos óseos asociados con este evento.

\section{Áreas de almacenamiento y materiales cerámicos relacionados a esta actividad: estructuras 4, 6 y 7}

Debido a que este estudio está relacionado con aspectos de almacenamiento prehispánico, se hará referencia a las estructuras 4, 6 y 7, cada una de estas relacionas íntimamente con este aspecto, ya que, de acuerdo con las interpretaciones, refieren a estas como áreas de almacenamiento o bodegas.

En el sitio arqueológico Joya de Cerén tenemos un claro ejemplo de almacenamiento, en su mayoría relacionado con la agricultura, aunque existe evidencia de diversificación de recursos como materiales líticos, minerales y almacenamiento vivo, descrito anteriormente y expresado en este sitio por la evidencia de un pato atado de una de sus extremidades, probablemente como muestra de domesticación. Además, son claras las evidentes zonas de cultivo y particularmente la gran disposición de los habitantes en la aldea.

Como ya sabemos, para el almacenamiento de materiales orgánicos perecederos como maíz y frijol, entre otros, deben existir actividades de producción (agricultura), recolección o intercambio previas, sin la existencia de estas en un nivel de especialización bastante alto no sería posible el almacenamiento de recursos y la diversificación que es evidente que hubo en Joya de Cerén.

\section{Estructura 4}

Ubicada en el actual complejo con el mismo número, evidencia una estructura hecha de una base de tierra apisonada con una dimensión de $3.2 \times 3.25$ m (10.4 m2) y una altura aproximada de $1.70 \mathrm{~m}$, cuatro columnas sólidas probablemente hechas de tierra apisonada sujetan 
4 paredes de bajareque, una de ellas colapsada por la inclemencia de la erupción volcánica (pared sur) y otra en el interior con dirección oeste-este, que divide la edificación en dos cuartos (norte y sur).

Según Sheets (2013), en el cuarto norte, el más pequeño, se registró un estante donde se colocaron varios artefactos, este estaba elaborado por varas de madera amarradas con doble cuerda. En cuanto al cuarto sur, el rasgo interno más predominante fue la localización de un granero circular (canasto o material especial para el almacenamiento) [figura 1] con un diámetro aproximado de $1 \mathrm{~m}$, el cual contenía mazorcas de maíz ya peladas. Sobre la base de este granero se habían colocado hojas perpendicularmente para aislar la humedad del maíz ya procesado, asimismo, se encontraron, aproximadamente, 83 mazorcas de maíz y el resto de un ratón, que probablemente se alimentaba del contenido.

Además de este hallazgo significativo, en la fachada de dicha estructura se había un metate con horquetas, donde, según los análisis químicos elaborados, se demostró el procesamiento de semillas de algodón, probablemente para extracción de su aceite para utilizarlo en la preparación de alimentos o como aglutinante para pigmentos.

Figura 1. Plano de registro descubierto en la Estructura 4 al momento de la excavación.

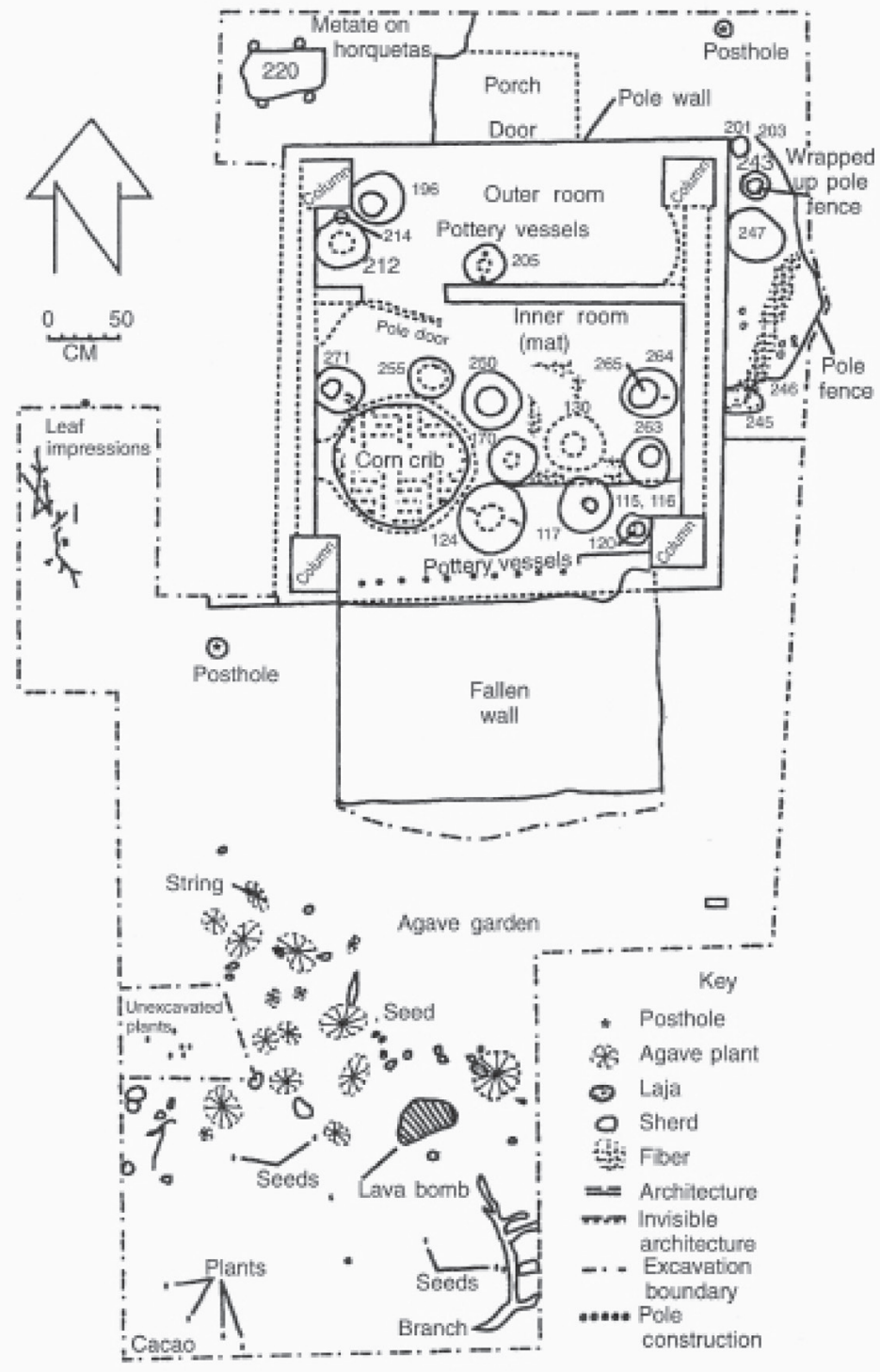


Según Sheets (2013), los artefactos, objetos portátiles y materiales orgánicos preservados en la Estructura 4 claramente indican su función como bodega, siendo lo interesante, además de esta función, que también era un área de trabajo, donde se procesaban hojas de agave para obtener fibras, así como para pintar y comer.

Con respecto a los materiales cerámicos que almacenaban, su mayoría se encontraba en el piso; una de estas contenía semillas de cacao. En total, 10 vasijas estaban con el estante, incluyendo un incensario con agarradero, dos vasijas polícromas, dos cuencos polícromos, dos cuencos trípodes polícromos, cuatro cántaros engobe raspado y un cuenco de boca abierta. Un cántaro estaba lleno de semillas de cacao y otro con material orgánico no identificado. El estante también tenía un pedazo de un tejido hecho de algodón, una aguja de hueso, un posible tapiscador de hueso y algún pigmento (Sheets, 2013:126).

Además, el cuarto sur contenía numerosos artefactos, algunos en el piso y otros dispuestos sobre muebles y estantes. Doce vasijas de cerámica estaban en el piso, de las cuales nueve eran del tipo Guazapa engobe raspado; seis no tenían un contenido identificable. De las tres con contenidos reconocibles, en dos había semillas de cacao y la otra tenía una mezcla de semillas de cacao con otras pequeñas redondas y nueces; y esta vasija aparentemente estaba cubierta con una tela de algodón elaborada en forma de gasa. Un roedor fue encontrado dentro de la vasija, lo que evidencia que la tela no fue suficiente para mantenerlo afuera. Otra vasija tenía la punta del cuerno de un venado, que pudo servir para desgranar maíz, así como todavía se hace en muchas áreas en Centroamérica (Sheets, 2013:128).

En resumen, según Sheets (2013), podemos interpretar que la función principal de la Estructura 4 era de almacenaje. Un rango impresionante de técnicas para almacenar granos se empleó aquí, incluyendo secar, colgar, uso de vasijas de cerámica, de granero, de redes suspendidas y canastas sobre el piso. Se almacenó una gran variedad de granos, incluyendo maíz, frijoles, cacao, algodón, guayaba, pipián y chiles, demostrando un alto grado de diversificación de recursos.

\section{Estructura 6}

Ubicada en el actual Complejo 1, evidencia una estructura por una plataforma muy baja elevada solo de 20 a $30 \mathrm{~cm}$ de la superficie circundante con una dimensión de $3.2 \mathrm{x}$
$3.2 \mathrm{~m}$ de un lado (10.24 m2), asimismo, las paredes de esta estructura eran una variante de bajareque, donde solamente se recubrieron las varillas en la parte baja a unos de 10 a 35 $\mathrm{cm}$, esto permitía la buena circulación del aire. Además, es probable que al momento de la erupción esta edificación estaba en proceso de construcción o renovación.

Figura 2. Plano de registro descubierto en la Estructura 6 al momento de la excavación.

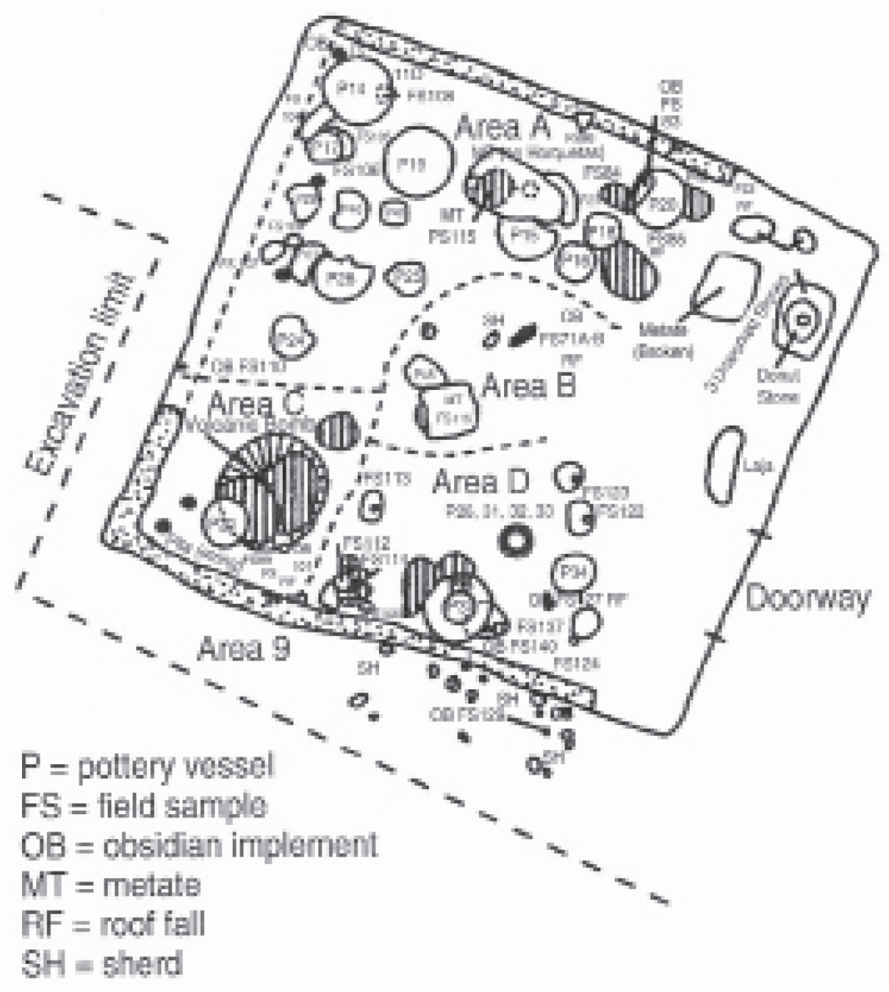

Fuente: Sheets, 2013

De acuerdo con sus rasgos, subdividieron la bodega en cuatro áreas, ya que existía un cierto patrón en los artefactos (figura 2). La primera, ubicada en el segmento norte, donde se encontró la variedad más grande de artefactos de todas las áreas. Es la más lejana a la puerta, y los artefactos habrían sido usados frecuentemente. Hay un denso almacenamiento de vasijas cerámicas (algunas llenas con semillas), morteros, artefactos de obsidiana, un metate y su mano de moler. El metate estaba montado en las horquetas muy bajas, probablemente utilizado por una persona de baja estatura. Un dato interesante es la identificación de cinco metates, 4 de ellos en sus horquetas y uno en el piso, del cual la mano se ubicó en la Estructura 11 (cocina), esto demuestra un alto procesamiento de granos probablemente de maíz en esta área que, si se contextualiza, también 
colinda con la Estructura 10, cuya función probablemente estaba desarrollada para organizar festejos o ceremonias.

Según Sheets, (2013) la parte norte del edificio tenía 18 vasijas de cerámica, de las cuales diez habían sido dañadas durante su uso, pero no habían sido descartadas. Había una alta frecuencia de cerámica decorada polícroma: 5 vasijas. Una de ellas tenía una forma ingeniosa para llenarse mediante un agujero del grueso de un lápiz. Alguien diseñó un tapón cilíndrico de un tiesto grueso y le dio la medida exacta del agujero. Algunas otras vasijas de mediano tamaño con asas aparentemente tenían agujeros hechos a propósito en los lados, pero la razón de esto es desconocida. Las cinco manos de morteros probablemente fueron usadas para la manufactura de instrumentos de lítica, como darle forma a metates, manos, piedras dona y otros implementos. La segunda área posee una mínima cantidad de artefactos, por ubicarse enfrente del acceso a la bodega. En este lugar estaba el grupo de navajas de obsidiana sin usar; y en el piso de esta área solamente se encontraron dos artefactos: un metate parcialmente volteado y una vasija, fragmentada pero en uso.

La tercera área no contenía gran cantidad de artefactos; y como dato interesante, en esta pequeña zona se registró una bomba volcánica que destruyó una vasija, dejándola en varios fragmentos esparcidos en el suelo. Restos de cuerda en esta vasija sugieren que estuvo colgada del techo.

Y finalmente, la cuarta área presentaba las mismas características de la primera, por el hecho que estaba llena de artefactos, algunos en el techo y otros en el piso. No es un área tan grande y está ubicada inmediatamente hacia la izquierda mientras uno entra al edificio. En esta área se encontró un fragmento de madera carbonizada con algunas marcas de cortes, cerca de la obsidiana que estaba guardada en el techo. Cortar madera para formar tablas largas y planas no era una actividad común en Joya de Cerén, pero cortar madera para objetos pequeños como postes, palos y vigas sí era común.

Los objetos identificados en esta bodega incluyen un malacate, pigmento rojo de hematina mezclado con mica en un contenedor orgánico, probablemente un morro. La mezcla de pintura roja y mica genera un color rojo brillante similar, pero no tan brillante como la hematita especular, que es rara y presumiblemente más cara, ya que está llena de cristales resplandecientes de óxido de hierro.

Al lado de esta mezcla, estaba una vasija Copador polícroma con "líneas de melón" para servir comida. Probablemente estaba en uso activo, fue encontrada dentro de la bodega. Cerca de ella estaba un cántaro del tipo Guazapa engobe raspado que pudo haber tenido líquido. Ambas vasijas son ejemplos de contenedores que estaban parcialmente quebrados, pero que seguían siendo usados, tal vez con algún cambio en sus funciones (Sheets, 2013). Junto a esta última, se identificaron cuatro vasijas guardadas cerca de la puerta; un incensario con un pedestal de base y un agarradero largo con una cara como decoración, además este demostró ser un posible patrón donde, en todas las estructuras domiciliares, bodega o cocina, tenían su propio incensario guardado. Descansando sobre el incensario había tres vasijas, dos Guazapa engobe raspado y una vasija del tipo Cashal. Estas tres vasijas pudieron ser usadas para ritos familiares, pero, aparte de su relación con el incensario, no hay otra evidencia de esto. Todas las vasijas estaban vacías al momento de la erupción.

Un poco más adentro de la bodega, había un cántaro grande del tipo Guazapa engobe raspado que estaba tapado por un cuenco hemisférico más pequeño. No se pudo identificar algún contenido dentro de las vasijas. Más allá de las dos vasijas había un mortero de mano, una piedra pómez para alisar y un pato, que posiblemente estaba vivo antes de la erupción, se encontraba amarrado de una de sus extremidades a la pared sur. En esta área de la bodega se encontraron tres piedras donas; una estaba en el piso cerca de la parte trasera del área cuatro. No había evidencia de un palo o algo parecido dentro de ella. Otras dos piedras donas estaban guardadas sobre la entrada y a casi un metro adentro del edificio en las vigas del techo.

\section{Estructura 7}

La Estructura 7 se ubica en el Complejo 2, con una dimensión de $3 \times 3$ metros (9 m2) y se encuentra muy cerca de la Estructura 2, poseía 4 paredes de bajareque, las cuales al momento de la erupción colapsaron todas hacia fuera y que originalmente el edificio tenía una entrada hacia el oeste, pero posteriormente fue remodelado con entrada al norte, $y$ la grada se convirtió en un asiento improvisado. 
Figura 3. Plano de registro descubierto en la Estructura 7 al momento de la excavación.

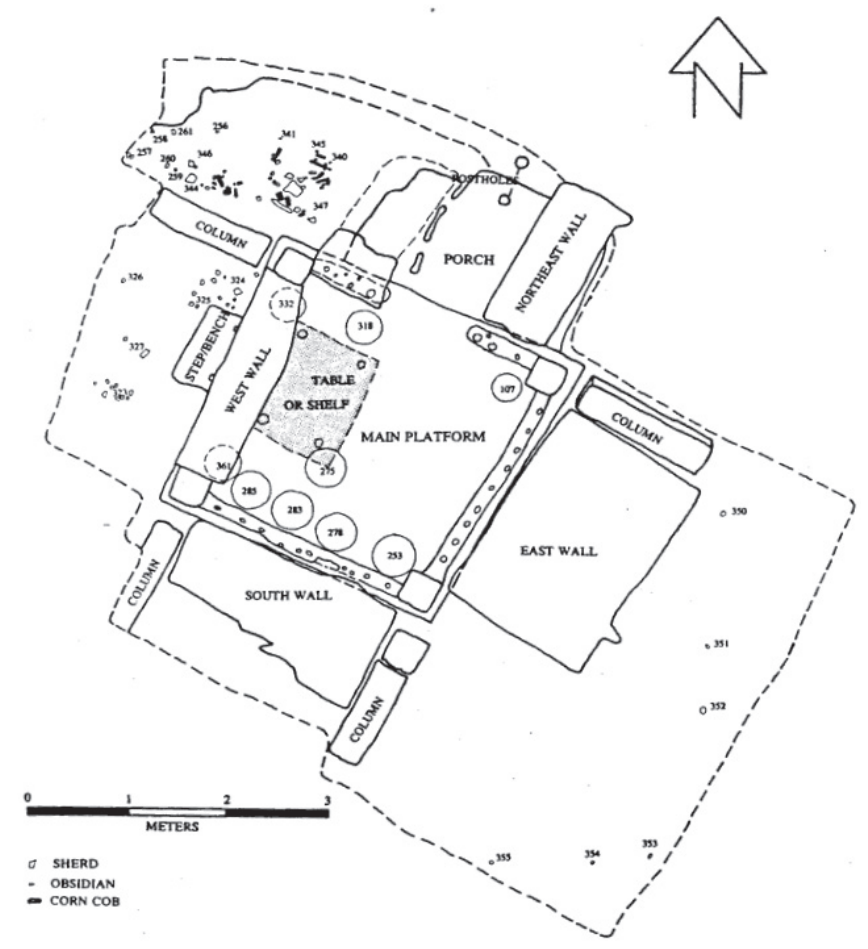

Fuente: Sheets, 2013

Sheets (2013) considera que la naturaleza y la densidad de los artefactos internos indican que esta era una bodega; una serie de cinco cántaros grandes de almacenaje estaban colocados sobre el piso, alineados en la pared trasera. La mayoría es del tipo Guazapa engobe raspado. No es común tener tantos cántaros de este tamaño vacíos; y ahora creemos que servían para proporcionar agua al baño sauna ubicado hacia el sur. Aparte de estos, sobre el piso también había una vasija de tamaño medio y dos vasijas grandes; guardaban varios tipos de semillas. Un sorprendente número de artefactos estaban suspendidos del techo dentro de la bodega. La mayoría de vasijas cerámicas estaban suspendidas en vez de estar descansando en el piso (figura 3). Es muy probable que, si todos los artefactos que estaban suspendidos del techo hubieran estado sobre el piso, el interior de esta bodega hubiera estado demasiado lleno de objetos para ser usado, evidenciando de esta manera la optimización del espacio.

Un grupo de cinco vasijas miniaturas, cuatro cuentas de jade y dos de otro material y cinco fragmentos de conchas, en la esquina suroeste, probablemente almacenadas en un recipiente orgánico (morro o piel); es posible que las cuatro cuentas y el pendiente pertenecieran a un collar, pero no se encontraron hilos o cuerdas. Estos son bienes de lujo que, junto con la arquitectura, indican un grado más alto de riqueza en el Complejo 2. Las vasijas miniaturas (perfumeras) poseen evidencia de pigmento rojo, y todas estaban decoradas. Según el análisis del pigmento, corresponde a sulfato de mercurio ( $\mathrm{HgS}$ ) conocido como cinabrio, mineral también de difícil adquisición y utilizado solo en actividades que estaban relacionadas con ceremonias $u$ otras actividades religiosas de la élite.

Como lo menciona Sheets (2013), otras nueve vasijas de cerámica o vasijas parciales se guardaban arriba, dentro de la bodega; dos probablemente en el estante y las otras en el techo. Estas incluyen un plato polícromo trípode con tres monos machos alrededor, dos cantaros medianos y dos platos. Una hachuela de piedra verde se encontró dentro de una de las vasijas grandes que estaba junto a la pared sur.

Una figurilla de hueso tallado con la forma de un hombre viejo con un sombrero alto se encontró en buenas condiciones, asimismo otro objeto orgánico importante: una nuez de palmera perforada por un palo de madera, formando un malacate y con su utensilio para hacerlo girar.

Otro material particular registrado en todas las bodegas son los hemisferios de ceniza de leña, producto probablemente relacionado con la recolección en cocinas del sobrante de ceniza de la leña utilizada para los alimentos. Es probable que este material sirviera para humedecer los granos de maíz antes de molerlos, ya que son demasiado duros de moler y, por lo tanto, necesitan ablandarse para suavizar la cáscara del grano, demostrando así una posible técnica incluso de almacenamiento, para su posterior producción y consumo.

\section{Discusión}

Como vimos en párrafos anteriores, según Hirth (2012), los sistemas de almacenamiento varían y se complejizan según avancen las culturas en sus modos de producción. Asimismo, de acuerdo con Hirth (2012), existen tres modelos teóricos para comprender el almacenamiento de sociedades antiguas: almacenamiento contra los riesgos de subsistencia, proyección social y economía política. El primero hace referencia a las estrategias que conlleva su ubicación de espacios con excedente de recursos, recolección, almacenamiento y consumo; el segundo, al 
almacenamiento como proyección social, que se remite al hecho de que, de acuerdo con los recursos almacenados por una sociedad, pueden otorgarle beneficios como poder o estatus, ya que tendrá más posibilidades de subsistencia, además, si estos son bienes diversos y se encuentran en lugares específicos o destinados para esta actividad; y por último, en relación con el almacenamiento como instrumento de inversión y de economía política, es relacionado directamente con sociedades políticamente organizadas, donde el almacenamiento juega un papel importante en el crecimiento de su influencia e interacción con otros pueblos.

Si empalmamos estas teorías en los contextos identificados en Joya de Cerén, sitio que evidencia las actividades cotidianas de una aldea maya del período clásico tardío sepultada por una erupción volcánica (volcán Loma Caldera ubicado a $1.5 \mathrm{~km}$ al noroeste del sitio), hecho que permitió tener un espacio de tiempo encapsulado de tan valiosa información, donde vemos reflejados aspectos como edificaciones con funciones específicas; sistemas constructivos: arquitectura (bajareque y tierra apisonada); áreas de cultivo: agricultura; alimentación y almacenamiento (viviendas y bodegas); actividades rituales (casa de la shamana y baño sauna); uso de espacios: posibles unidades domésticas y plaza; cerámica y lítica características del sitio y evidencia del impacto de un fenómeno natural (erupción volcánica) sobre las viviendas. Todos estos datos pueden ser estudiados y discutidos con las teorías antes mencionadas y así tener una mejor comprensión de la complejidad social de Joya de Cerén.

\section{Análisis de la cerámica procedente de las estructuras 4,6 y 7}

Numerosos artefactos y materiales orgánicos han sido rescatados de estas edificaciones en Joya de Cerén, identificando tipos cerámicos característicos de las fases Xocco y Payu descritas por Sharer (1978), y tipos de cerámica pertenecientes al Valle de Zapotitán descritas por Beaudry (1983), todas estas características indiscutiblemente son el período clásico tardío. Asimismo, coincidiendo con los datos arrojados por los análisis de datación.

Como lo mencionamos anteriormente, estas edificaciones corresponden, de acuerdo con sus contextos y materiales ahí encontrados, a áreas destinadas al almacenamiento, que en aspectos generales podemos mencionar que fue muy diverso: desde materiales para subsistencia (procedentes de la agricultura y recolección) hasta para la elaboración de herramientas, para la producción de alimentos (metates, manos de metate, piedras donas, navajas prismáticas de obsidiana, entre otros); materiales producto de talleres de manufactura como, las fibras vegetales para elaboración de cuerdas, y materiales suntuosos como jade y cinabrio.

Cabe mencionar que la cerámica en estas tres edificaciones jugó un papel importante en el almacenamiento de materiales diversos, en su mayoría orgánicos, dejando claro que esta era destinada a este uso, quedando descartado el uso de fosas subterráneas, como anteriormente se menciona en otros sitios arqueológicos en Mesoamérica, lo que explicaría cómo las evidencias encontradas, tanto en Cahokia, en Estados Unidos, como en Teotihuacán, en México, que la aldea de Joya de Cerén no necesitaba del almacenamiento de fosas, ya que tenía almacenamiento superficial, el cual no estaba en riesgo, además de la evidencia de restos materiales suntuosos como jade, hachuelas de piedra verde, perfumeras (vasijas miniaturas encontradas en la Estructura 7) y pigmentos como cinabrio, hematita y mica sugieren que Joya de Cerén no era una aldea común, más bien tienen un alto estatus, que se concibe como una aldea común, donde el almacenamiento de estos recursos sería completamente reducido e incluso fuera de un carácter comunal, no como una concepción probablemente privada donde cada área habitacional, como el caso de la Estructura 1 y 2, posee junto con estas bodegas para almacenamiento (Estructuras 6 y 7), dotando a estas áreas habitacionales de beneficios y un alto grado de subsistencia. Este planteamiento puede ser sustentado desde la teoría del almacenamiento como proyección social, generando de esta manera un nuevo paradigma para la compleja comunidad de Joya de Cerén.

Se ha identificado, además, que los tipos cerámicos más comunes en estas edificaciones especializadas en el almacenamiento del sitio son del tipo Guazapa engobe raspado, Copador, Cashal, Gualpopa, Campana (Sheets, 2002: 121) y otros tipos polícromos característicos de las fases cerámicas antes descritas. Es interesante destacar que estos tipo cerámicos fueron identificados en diferentes formas como cántaros, cuencos semiesféricos, incensarios, ollas, platos trípodes, como se evidencia en el la figura 4, vajilla y tipos cerámicos muy variados; que generalmente este fenómeno es evidente en sitios ceremoniales o de élite para sitios contemporáneos, siendo uno de ellos muy cercano a Joya de Cerén: San Andrés (figura 5). 
Figura 4. Cuadro descrito por Sheets (2002) donde se compara los materiales cerámicos identificados en las estructuras 4,6 y 7

firing very large vessels. Such vessels must be built over a period of days, so that the wall dries enough to support the upper parts; the size and wall thick. ness require special handiling and cartét controt of the firing condittons, Imaginative Tepresentations of elfigies bespeak artisisal skit; the caiman pot and other effigy vessets also

Cream slip is a favored mode of decoration at Cerén, used on the Guazapa Ceramic Group in variety of ways across a wide range of forms and sizes. Whitic crean slp is found during tils time

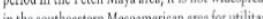

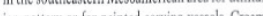

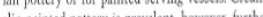
stip painted potery is prevalent, towcre, tarther south in the Gitater Nicoya zonc, subgrting a d. tion. Interesting shape and decorative characteristic were noted among the design painted resses. Th.

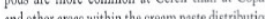
thl het as we

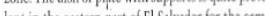
lent in the est hom time perial, Honce. Thas, we mas have an ample of a fontict bend gor

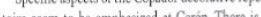
toire seem to be che dorinn instance. The clongated simm in figure mot.

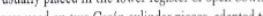

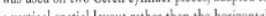

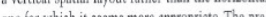
one for whit setms mor apropiate. The pa sention

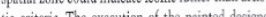

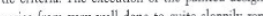

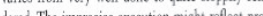
dere

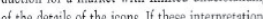

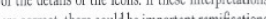
art cout the ethnic ident tity uf the roup acouiring the the the

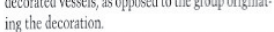

Certain ceramics are rare or absent. There is very little Campana Fine Line Polychrome, a quite com. mon type at other western El Salvador sites. The few pieces at Cerén verify that the cruption-period accupation coincided with the enoch when Cam. pana was being made. No ready explanation can be given for the Camnana searcity, but it annears the the Cerén community was more closely connected
UDRY-CORBETT, WITH CONTRIBUTIONS BY BISHOH

vith the cream paste distribution sphere than with hat assocateded with the Campana Group ceramic A rotabie a assence in the etuption.period Certh pottry copus is the comal cook . comal in the rightlands and ts absact fron the

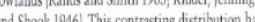

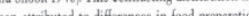

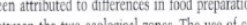
arsimlies the consumtion of tort tlas the lask onlies the consumption of tamales or ot ther non piddle-prepared foods Comales are quite commo

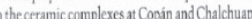
rom the Early Classic Period but are an infecequent

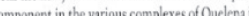

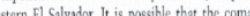
al sila dis ponte that the com

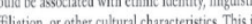

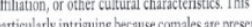

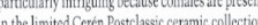
the Inventories

It is hypothesized that all households engaged in the same basic set of sustenance events, that is ansie, storage, processing, preparation, consump Lon, and disposal of foodstuffs. It also is though hat similar goods and objects were involved in those events-commodittes (foodstuffs), contain es ( pottery, baskets, gourds, nets), facilities work saces or features such as mats, tables, shelves, hor. uetas forked props, hearths, and implements on tols (grinding stones, donut stones, chipped ston vols. The amount of time devoted to sustenance ctuvties, as well as the location of these actuinties, could be more variable, relating to aspects of houss. fold composition and the domestic unit's rol. within the community

STOREHOUSE INVENTORIES

torehouses were loci for keeping perishable com. modities as well as imperishable household imments and craft supplies. Food processing vern kely also took place in these structures. Since e have excavated three storchouse structures, ce. mic inventory data from that provenience cate ry can be compared among houscholds

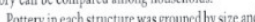
shape into categories reflecting general functions
CERAMICS AND THEIR USE AT CERÉN

I2I

TABLI 13.3. Potery Inrentern, Storehouss

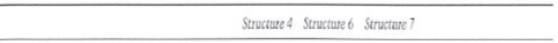

Complete vessels'

Utitar Small |less than $12 \mathrm{~cm}$ Small-Medium (12-15 Medium $16-19 \mathrm{~cm})$

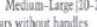

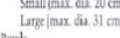
Open with hundeflicaured with hantlo

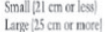

Panted sering versels

Recurred bonks

Cylinder resc

Dushes tripod and posibile tripod

Other vessels

Incensaring/censers
Slipped incured bools Minitures

Total, complete ress:

Partial vesecels

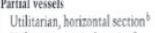

Utilitarian, verical sectina ${ }^{2}$

Serving, horizantal sectios

Serving, vertical sction
Unclussifed brizonal scction

Total, partial resids

Grand total, all rased?

Paitred ressels classificd by trpe (vidole and partial|)

Copalor

Guapopia

Campana
Unclassfied

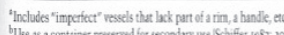
cantain-od dolders, or derer tio

maximum diameter, beight, and neck and handle bouses demonstrate community-wide sustenance detalls were made. Associations among these vari- practics and religious activity. The total nurber of ables were noted to the point where it was pos. vessels per storehouse is similar(Table 133. When sible to assign jars, for instance, to size groupings not in use, potery generally was stored similarly. if two measurements could be made In addition lars usually were placed directly on the floet to the size-shape analysis volume capacity poten- Painted serving dishes were kept elecentere - 0 a a

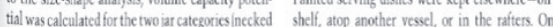
with handles, necked without handless, using the exception was in Structure 4, where three round formula given in Ericson and Strickel 1973. Pottery inirentory similarities among the store. ner removed from general traffic Each storchouse 
Figura 5. Materiales cerámicos procedentes de las estructuras 4, 6 y 7.

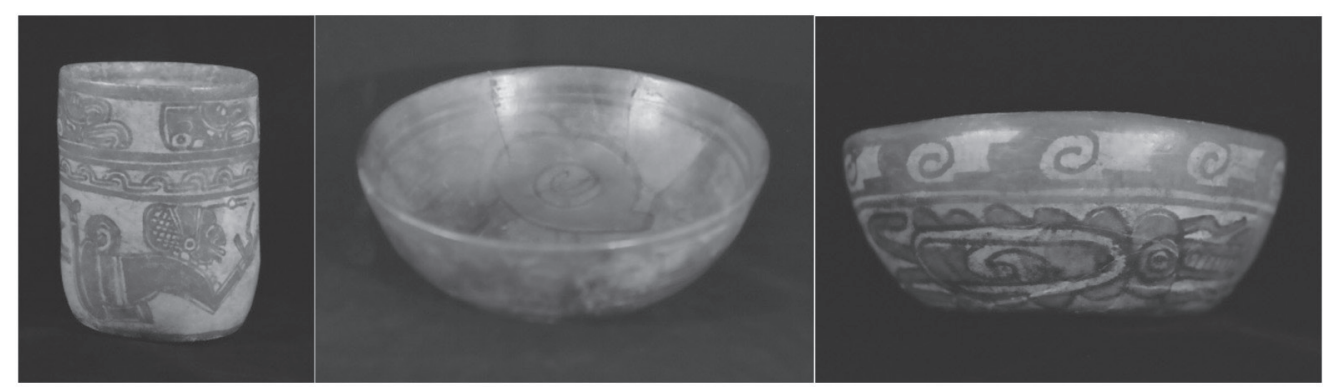

Cerámica procedente de la Estructura 4
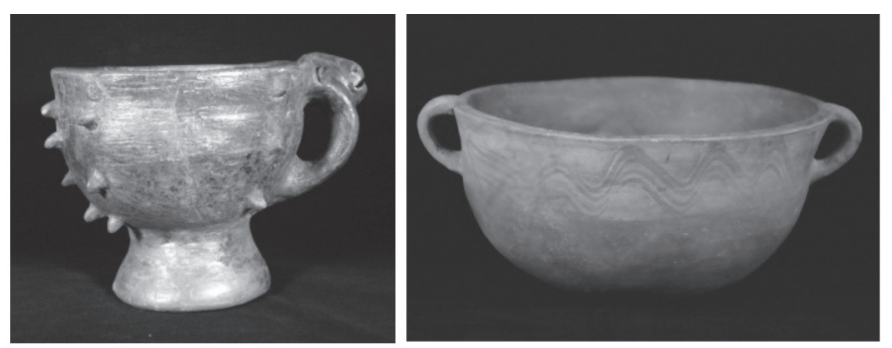

Cerámica procedente de la Estructura 6
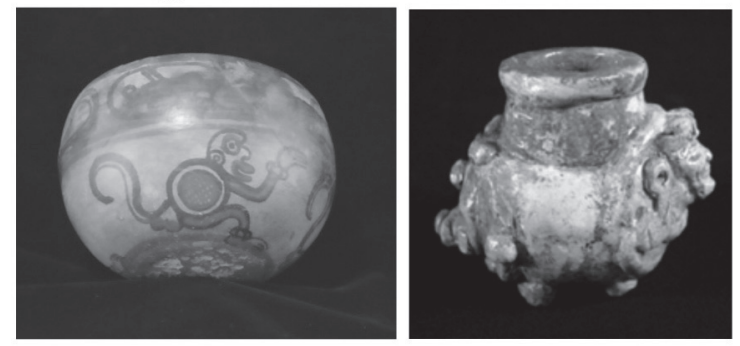

Cerámica procedente de la Estructura 7

Fuente: Sheets, 2002

Incluso vasijas miniatura (perfumeras) [figura 6], identificadas en la Estructura 7, evidencian el uso de estas para fines probablemente relacionados con la manufactura de cerámica u otras actividades en las que se utilizaban pigmentos. Estas, a la vez, demuestran que su manufactura requiere de una especialización, ya que probablemente fueron elaboradas a partir de moldes y así lograr finos detalles en una pieza tan pequeña.

Figura 6. Fotografías de vasijas miniaturas (perfumeras) encontradas en la Estructura 7.
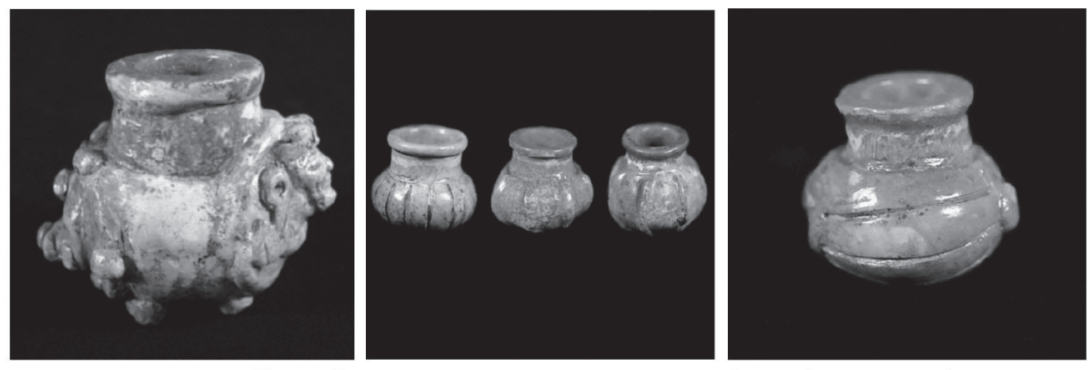

Fuente: Sheets, 2002 
Dado a que las piezas cerámicas encontradas en las estructuras 4, 6 y 7 fueron utilizados exclusivamente para almacenar, tanto materiales perecederos (granos producto de la agricultura y la recolección) como inorgánicos (pigmentos), podemos interpretar que las actividades relacionadas con el almacenamiento en Joya de Cerén eran muy complejas y diversificadas, así como también que el uso de un tipo cerámico no está relacionado intrínsecamente a una función, sino más bien a su forma; y de esta manera guardar alimentos o materias primas para utilizar en el día a día o por un tiempo indeterminado.

Asimismo, es interesante el hecho de que en Joya de Cerén no se hayan registrado hasta el momento formaciones troncocónicas o fosas subterráneas para almacenamiento, que son características de comunidades domésticas probablemente de bajo estrato social, lo cual descartaría por el momento, ya que aún falta hacer más investigación arqueológica, que el sitio sea una aldea común, más bien, de acuerdo con los materiales registrados en estas tres bodegas, se demuestra que en conjunto tienen muchos rasgos característicos o asociados con personajes de élite o que poseían por lo menos un estatus alto en una sociedad compleja como las que se desarrollaron en el período clásico tardío en Mesoamérica, acompañando a esto los hechos de que estas bodegas tienen un espacio destinado especialmente para el almacenamiento, y que cada una posea un promedio de $9.88 \mathrm{~m}^{2}$ destinados solo para esta función, aunque, de acuerdo con los rasgos identificados en donde se plantea que el almacenamiento era aéreo o suspendido del techo para maximizar el espacio, esto podría sugerir, incluso, el triple de espacio utilizado para el almacenamiento en Joya de Cerén.

La diversificación de alimentos, minerales y otros materiales orgánicos demuestra la complejidad social, tanto de estrategias organizativas, técnicas de almacenamiento y conservación de los bienes como de producción y procesamiento de los bienes que poseía Joya de Cerén al momento de la erupción volcánica, no dejando de lado los materiales orgánicos en las milpas que aún hacía falta de procesar y almacenar. Por tal razón será necesario ahondar más sobre tan importante actividad en este sitio y seguir generando descubrimiento como el planteado en este estudio, el cual es de vital importancia para la comprensión de los pueblos originarios que en el pasado habitaron el actual territorio salvadoreño.

\section{Referencias}

Bortot S. \& Michelet, D., Darras, V. (2012). "Almacenamiento prehispánico del Norte de México al Altiplano Central". México. Centro de Estudios Mexicanos y Centroamericanos: Universidad Autónoma de San Luis de Potosí.

Boudry, M. (1983). "The ceramics of the Zapotitan Valley". En Archeology and Vulcanism In Central America, The Zapotitan Valley of El Salvador (pp. 161-190). Austin. University of Texas Press.

Emery, K., \& Gotz, C. (2014). La Arqueología de los Animales de Mesoamérica (1st ed.). Atlanta: Lockwood Press.

Guevara, S.A. (2012). "Algunas formas prehispánicas de almacenamiento en el norte de México". En S. Bortot, D. Michelet, \&V. Darras, Almacenamiento prehispánico del norte de México al altiplano central (pp. 73-76). México: Universidad Autónoma de San Luis Potosí.

Hirth, K.G. (2012). "La modelización del almacenamiento prehistórico: subsistencia, desigualdad y complejidad política". En S. Bortot, D. Michelet, \& V. Derras, Almacenamiento prehispánico del norte de México al altiplano central (pp. 13-26). México. San Luis Potosí: Centro de Estudios Mexicanos y Centroamericanos.

Manzanilla, L. (2012). "El almacenamiento en Teotihuacan: enfoques metodológicos". En S. Bortot, D. Michelet, \& V. Derras, Almacenamiento prehispánico del norte de México al altiplano central (pp.73-80). Mexico. San Luis Potosí: Centro de estudios Mexicanos y Centroamericanos.

Sharer, R. (1978). The prehistory of Chalchuapa, El Salvador. Vol. 3 Pottery and Conclusions. Pennsylvania. University of Pennsylvania Press.

Sheets, P. (2002). Before the Volcano Erupted, The Ancient Village in Central America. United States of America. University of Texas Press. Primera edición.

Sheets, P., \& Gallardo, R. (2013). Joya de Cerén: Patrimonio de la Humanidad 1993-2013. El Salvador. Editorial Universitaria, Universidad de El Salvador. Primera edición.

Uruñuela, G., Guevara, \& Plunket, P. (2012). “De troncocónicos a cuexcomates". En S. Borot, D. Michelet, \& V. Darras, Almacenamiento prehispánico del norte de México al Altiplano central (pp. 42-60). México: Universidad Autónoma de San Luis Potosí. 